JOURNAL OF DIFFERENTIAL EQUATIONS 4, 661-679 (1968)

\title{
The Bifurcation of One or More Closed Orbits from an Equilibrium Point of an Autonomous Differential System*
}

\author{
Nathaniel Chafee \\ University of Michigan, \\ Ann Arbor, Michigan 48106
}

Received September 13, 1967

\section{INTRODUCTION}

In the present paper we will consider an autonomous ordinary differential equation of the form

$$
\dot{x}=P(\epsilon) x+X(x, \epsilon),
$$

where $x$ and $X$ are real $n$-vectors in the Euclidean space $E^{n}(n>2), \epsilon \geqslant 0$ is a small parameter, $P$ is a real $n \times n$ matrix, and $X(0, \epsilon)=0, \epsilon \geqslant 0$, so that the origin $x=0$ is an equilibrium point of $(*)$ for $\epsilon \geqslant 0$. We will require that both $P$ and $X$ be continuous in their respective arguments and that $X$ satisfy a suitable Lipschitz condition in $x$, explicitly stated below, from which it follows that $|X(x, \epsilon)|=o(|x|)$ as $|x| \rightarrow 0$. Furthermore, we will assume that for each $\epsilon \geqslant 0$ there exist two distinct complex-conjugate eigenvalues of $P(\epsilon)$ which have zero real part for $\epsilon=0$ and positive real part for $\epsilon>0$. The other $n-2$ eigenvalues of $P(\epsilon)$, we will assume, have negative real parts for all $\epsilon \geqslant 0$. Finally, we will suppose that when $\epsilon=0$ the equilibrium point at the origin $x=0$ is asymptotically stable in the sense of Liapunov.

The object of our investigations is to obtain a complete description of the qualitative behavior of solutions of $(*)$ near the origin when $\epsilon>0$. Our principal results can be described as follows.

For $\epsilon>0$ and sufficiently small, there exist for $(*)$ two closed orbits $\gamma_{1}(\epsilon)$ and $\gamma_{2}(\epsilon)$, not necessarily distinct, and as $\epsilon \rightarrow 0+$ these orbits uniformly contract to the origin. In this sense we can say that $\gamma_{1}(\epsilon)$ and $\gamma_{2}(\epsilon)$ bifurcate

* This research was partially supported by the National Science Foundation (Grant No. GP-4632) and the U. S. Army Research Office, Durham (Contract No. DA-31-124-ARO-D-270) as part of the author's doctoral thesis written under the direction of Professor Jack K. Hale at Brown University in 1965-66, and by the U. S. Air Force Office of Scientific Research (Grant No. 942-65) at the University of Michigan, Ann Arbor, in 1967. 
from the origin as $\epsilon$ increases from zero. The orbits $\gamma_{1}(\epsilon)$ and $\gamma_{2}(\epsilon)$ lie on a local integral manifold $M^{2}(\epsilon)$ homeomorphic to a two-dimensional open disk and containing the origin $x=0$; regarded as closed Jordan curves in $M^{2}(\epsilon)$, $\gamma_{1}(\epsilon)$ and $\gamma_{2}(\epsilon)$ are concentric about the origin. In a certain $n$-dimensional neighborhood of the origin the solutions of $(*)$ are divided into two classes. The first class consists of solutions which, as $t \rightarrow+\infty$, approach the closed invariant set $\Omega(\epsilon)$ in $M^{2}(\epsilon)$ bounded by $\gamma_{1}(\epsilon)$ and $\gamma_{2}(\epsilon)$ (when $\gamma_{1}(\epsilon)=\gamma_{2}(\epsilon)$ this set degenerates into a single closed orbit of $(*))$, and the second class consists of solutions which approach the origin $x=0$ as $t \rightarrow+\infty$. These latter solutions fill a local integral manifold homeomorphic to an $(n-2)-$ dimensional open ball.

Problems similar to the one to be treated in this paper have been studied by Andronov ([I], pp. 217-224), Hopf [7], and Brushinskaya [3] under hypotheses different from our own and with correspondingly different results. In particular, under the conditions assumed by these authors the invariant set $\Omega(\epsilon)$ is always a single closed orbit. An example is given below showing that under our own hypotheses such is not always the case.

\section{Statement of Hypotheses and Main Theorem}

Notation. Throughout this paper we will let $E^{n}$ denote the real $n$ dimensional Euclidean space and $j \cdot 1$ the Euclidean norm on this space. For any $r>0$ we will let $B^{n}(r)$ denote the open ball $\left\{x \in E^{n}: 0 \leqslant|x|<r\right\}$.

We consider an autonomous differential equation of the form

$$
\dot{x}=P(\epsilon) x+X(x, \epsilon),
$$

where $x$ and $X$ vary in some real Euclidean space $E^{n}(n>2), \epsilon \geqslant 0$ is a small parameter, and $P$ is a real $n \times n$ matrix. Henceforth, we shall assume that (1.1) conforms to the following hypotheses.

$\left(\mathrm{H}_{1}\right)$ There exist numbers $r_{0}>0$ and $\epsilon_{0}>0$ such that $P$ is continuous on the closed interval $\left[0, \epsilon_{0}\right]$ and $X$ is continuous on the domain $B^{n}\left(r_{0}\right) \times\left[0, \epsilon_{0}\right]$.

$\left(\mathrm{H}_{2}\right)$ For each $\epsilon$ in $\left[0, \epsilon_{0}\right]$ we have $X(0, \epsilon)=0$ so that the origin $x=0$ is an equilibrium point of (1.1).

$\left(\mathrm{H}_{3}\right)$ For each $r$ in $\left[0, r_{0}\right]$ there exists a $k(r)>0$ such that on the domain $B^{n}(r) \times\left[0, \epsilon_{0}\right]$ the function $X$ is uniformly Lipschitzian in $x$ with Lipschitz constant $k(r)$; moreover, $k(r) \rightarrow 0$ as $r \rightarrow 0$.

$\left(\mathrm{H}_{4}\right)$ For each $\epsilon$ in $\left[0, \epsilon_{0}\right]$ the mațix $P(\epsilon)$ has a complex-conjugate pair 
of eigenvalues $a(\epsilon) \pm i b(\epsilon)$ whose real and imaginary parts satisfy the conditions

$$
\begin{gathered}
a(0)=0, \quad a(\epsilon)>0 \quad\left(0<\epsilon \leqslant \epsilon_{0}\right), \\
b(\epsilon)>0 \quad\left(0 \leqslant \epsilon \leqslant \epsilon_{0}\right) .
\end{gathered}
$$

The other eigenvalues $\lambda_{1}(\epsilon), \lambda_{2}(\epsilon), \ldots, \lambda_{n-2}(\epsilon)$ of $P(\epsilon)$ have their real parts negative for all $\epsilon$ in $\left[0, \epsilon_{0}\right]$.

$\left(\mathrm{H}_{5}\right)$ For $\epsilon=0$ the equilibrium point of (1.1) at the origin is asymptotically stable in the sense of Liapunov (Lefschetz [9], p. 89).

Hypotheses $\left(\mathrm{H}_{1}\right)$ and $\left(\mathrm{H}_{3}\right)$ are sufficient to guarantee the usual properties of existence, uniqueness, and continuity in initial conditions for solutions of (1.1). In that which follows the solution of (1.1) assuming a given initial value $x_{0}$ at $t=0$ will be denoted by $x\left(t, x_{0}, \epsilon\right)$. In connection with this notation we should mention the well-known autonomous property of (1.1): the solution of (1.1) assuming a given initial value $x_{0}$ at a specified value of $t$, say $t_{0}$, is given by $x\left(t-t_{0}, x_{0}, \epsilon\right)$.

The results to be obtained in this paper can be summarized in the following manner.

THEOREM 1.1 Let (1.1) satisfy the hypotheses $\left(\mathrm{H}_{1}\right)$ through $\left(\mathrm{H}_{5}\right)$ and let $r_{0}$ and $\epsilon_{0}$ be as in $\left(\mathrm{H}_{1}\right)$. Then, there exist numbers $r_{1}, r_{2}$, and $\epsilon_{1}$ such that $0<r_{2} \leqslant r_{1} \leqslant r_{0}, 0<\epsilon_{1} \leqslant \epsilon_{0}$, and such that the following assertions are true.

(i) For each $\epsilon \in\left(\epsilon, \epsilon_{1}\right]$ there exist for Eq. (1.1) two closed orbits $\gamma_{1}(\epsilon)$ and $\gamma_{2}(\epsilon)$ (not necessarily distinct) which lie inside a neighborhood of the form $B^{n}(r(\epsilon))$, where $0<r(\epsilon) \leqslant r_{2}$ and $r(\epsilon) \rightarrow 0$ as $\epsilon \rightarrow 0+$. Moreover, $\gamma_{1}(\epsilon)$ and $\gamma_{2}(\epsilon)$ lie on a local integral manifold $M^{2}(\epsilon)$ homeomorphic to an open disk in $E^{2}$ and containing the origin $x=0$. Regarded as closed Jordan curves in $M^{2}(\epsilon)$, $\gamma_{1}(\epsilon)$ and $\gamma_{2}(\epsilon)$ are concentric about the origin with, say, $\gamma_{1}(\epsilon)$ inside $\gamma_{2}(\epsilon)$ when these curves are distinct.

(ii) For each $\epsilon \in\left(0, \epsilon_{1}\right]$ that part of $M^{2}(\epsilon)$ which lies inside $\gamma_{1}(\epsilon)$ is filled by solutions of (1.1) which approach the origin as $t \rightarrow-\infty$ and which, except for the equilibrium point ar $x=0$, approach $\gamma_{1}(\epsilon)$ as $t \rightarrow+\infty$. No other solutions of $(1.1)$ remain in $B^{n}\left(r_{1}\right)$ for all $t<0$.

(iii) For each $\epsilon \in\left(0, \epsilon_{1}\right]$ that part of $M^{2}(\epsilon)$ lying outside $\gamma_{2}(\epsilon)$ but contained in $B^{n}\left(r_{2}\right)$ is filled by solutions of (1.1) which remain in $M^{2}(\epsilon) \cap B^{n}\left(r_{1}\right)$ for all $t>0$ and which approach $\gamma_{2}(\epsilon)$ as $t \rightarrow+\infty$.

(iv) For each $\epsilon \in\left(0, \epsilon_{1}\right]$ there exist solutions of (1.1) which approach the 
origin $x=0$ as $t \rightarrow+\infty$ and these solutions fill a local integral manifold $M^{n-2}(\epsilon)$ homeomorphic to an open ball in $E^{n-2}$ and containing the origin $x=0$.

(v) If for a given $\epsilon \in\left(0, \epsilon_{0}\right], x\left(t, x_{0}, \epsilon\right)$ is a solution of (1.1) for which $x_{0} \in B^{n}\left(r_{2}\right)$, then $x\left(t, x_{0}, \epsilon\right)$ remains in $B^{n}\left(r_{1}\right)$ for all $t>0$. Moreover, if $x\left(t, x_{0}, \epsilon\right) \nrightarrow 0$ as $t \rightarrow-\infty$ (see (iv)) then as $t \rightarrow+\infty x\left(t, x_{0}, \epsilon\right)$ approaches the closed invariant set $\Omega(\epsilon)$ consisting of those points in $M^{2}(\epsilon)$ which lie on $\gamma_{1}(\epsilon)$ or $\gamma_{2}(\epsilon)$ or between them. The solutions which approach $\Omega(\epsilon)$ contain in their positive-limiting sets one or more closed orbits (which may or may not coincide with $\gamma_{1}(\epsilon)$ or $\left.\gamma_{2}(\epsilon)\right)$.

The question arises, is it possible to improve the above results by showing that for all $\epsilon \in\left(0, \epsilon_{1}\right] \gamma_{1}(\epsilon)$ and $\gamma_{2}(\epsilon)$ coincide? Brushinskaya's work [3] indicates such a possibility. She has considered an equation of the form (1.1) satisfying $\left(\mathrm{H}_{1}\right)$ through $\left(\mathrm{H}_{4}\right)$ with the additional requirement that $X$ be analytic in $X$. In place of $\left(\mathrm{H}_{5}\right)$ she has imposed a condition on the sign of a certain parameter constructed from $H$. From this condition there follows $\left(\mathrm{H}_{5}\right)$ and results analogous to Theorem 1.1 except that $\gamma_{1}(\epsilon)$ and $\gamma_{2}(\epsilon)$ are replaced by a single stable limit cycle. Thus, we may ask whether or not Brushinskaya's conclusion holds under our more general hypotheses. The following example shows that this is not the case.

Consider the system of differential equations

$$
\begin{aligned}
& \dot{r}=r(r-\epsilon)^{2}(2 \epsilon-r), \quad(\epsilon \geqslant 0) \\
& \dot{\theta}=1, \quad z=-z, \quad
\end{aligned}
$$

where $r$ and $\theta$ are to be regarded as polar coordinates of a vector $y$ in $E^{2}$ and where $z$ is to vary in $E^{1}$. Letting $x=(y, z), x \in E^{3}$, we can easily transform (1.2) into a system of the form (1.1) and just as easily, we can verify that this system satisfies $\left(\mathrm{H}_{1}\right)$ through $\left(\mathrm{H}_{5}\right)$. Now, for each $\epsilon>0$ (1.2) has exactly two closed orbits $\gamma_{1}(\epsilon)$ and $\gamma_{2}(\epsilon)$ which are the circles in the $y$-plane about the origin having radii $\epsilon$ and $2 \epsilon$ respectively. It is easy to see that these closed orbits have the properties required of $\gamma_{1}(\epsilon)$ and $\gamma_{2}(\epsilon)$ by Theorem 1.1, with $M^{2}(\epsilon)$ here being the entire $y$-plane. Thus, Theorem 1.1 can not be improved in the sense indicated above.

On the basis of $\left(\mathrm{H}_{4}\right)$ and the continuity of $P$ in $\epsilon$, we may assume that (1.1) has the form

$$
\begin{gathered}
\dot{y}=A(\epsilon) y+Y(x, \epsilon), \\
\dot{z}=C(\epsilon) z+Z(x, \epsilon),
\end{gathered}
$$

where $y \in E^{2}, z \in E^{n-2}, A$ and $C$ are real matrices having for each $\epsilon \in\left[0, \epsilon_{0}\right]$ eigenvalues $a(\epsilon) \pm i b(\epsilon)$ and $\lambda_{1}(\epsilon), \lambda_{2}(\epsilon), \ldots, \lambda_{n-2}(\epsilon)$ respectively, and $Y$ and $Z$ are the $y$ - and $z$-components of $X$. That such an assumption is valid follows 
from standard arguments. Our notation for solutions of (1.3) is the same as that already indicated for solutions of (1.1) with the added convention that the $y$ - and $z$-components of $x\left(t, x_{0}, \epsilon\right)$ will be denoted by $y\left(t, x_{0}, \epsilon\right)$ and $z\left(t, x_{0}, \epsilon\right)$. In that which follows we will prove Theorem 1.1 for equations (1.3) rather than equation (1.1).

\section{The Existence of the Local Integral Manifold $M^{2}(\epsilon)$}

In this section we will prove the existence of the manifold $M^{2}(\epsilon)$ in which, according to Theorem 1.1, we expect to find the closed orbits $\gamma_{1}(\epsilon)$ and $\gamma_{2}(\epsilon)$. Moreover, we will derive certain properties of $M^{2}(\epsilon)$ crucial to proving in Section 3 the existence of $\gamma_{1}(\epsilon)$ and $\gamma_{2}(\epsilon)$. The techniques we will use here are modifications of techniques used by Bogoliubov and Mitropolsky [2], Likova [10], [11], and Hale [6] in their studies of integral manifolds. The results which are relevant to the proof of Theorem 1.1 are stated in Theorem 2.3 and its corollaries.

We begin by performing a number of constructions. From $\left(\mathrm{H}_{4}\right)$ there follows the existence of constants $\alpha>0$ and $K>0$ such that

$$
\begin{array}{lr}
\left|e^{C(\epsilon) t}\right| \leqslant K e^{-\alpha t} & \left(0 \leqslant t \leqslant \infty, 0 \leqslant \epsilon \leqslant \epsilon_{0}\right), \\
\left|e^{A(\epsilon) t}\right| \leqslant K e^{-(\alpha / 2) t} & \left(-\infty<t \leqslant 0,0 \leqslant \epsilon \leqslant \epsilon_{0}\right) .
\end{array}
$$

Next, referring to $\left(\mathrm{H}_{3}\right)$, we choose $\rho_{0} \in\left(0, r_{0}\right]$ so that

$$
k \stackrel{\text { defn. }}{=} k\left(\rho_{0}\right) \leqslant \frac{\alpha}{16} \min \left\{(K+1)^{-1}, K^{-2}\right\} .
$$

For $\rho_{0}$ so chosen and for $Y$ as in (1.3) we define a function $Y_{0}$ on the domain

$$
D=\left\{(y, z, \epsilon): 0 \leqslant|y|<\infty, 0 \leqslant|z|<\rho_{0}, 0 \leqslant \epsilon \leqslant \epsilon_{0}\right\}
$$

by setting

$$
Y_{0}(y, \boldsymbol{s}, \epsilon)=\left\{\begin{array}{lll}
Y(y, z, \epsilon) & \text { if } & 0 \leqslant|y| \leqslant \rho_{0} \\
Y\left(y \rho_{0} /|y|, z, \epsilon\right) & \text { if } & \rho_{0} \leqslant|y|<\infty
\end{array}\right\} .
$$

In the same way we define a function $Z_{0}$ on $D$ using $Z$ in (1.3). By means of $\left(\mathrm{H}_{1}\right),\left(\mathrm{H}_{2}\right)$, and $\left(H_{3}\right)$, it is easy to show that $Y_{0}$ and $Z_{0}$ are both continuous on $D$ and that on this domain they satisfy the inequalities

$$
\begin{gathered}
\left|Y_{0}(y, z, \epsilon)\right| \leqslant 2 k \rho_{0},\left|Z_{0}(y, z, \epsilon)\right| \leqslant 2 k p_{0} \\
\left|Y_{0}(y, z, \epsilon)-Y_{0}(\bar{y}, \bar{z}, \epsilon)\right| \leqslant k(|y-\bar{y}|+|z-\bar{z}|), \\
\left|Z_{0}(y, z, \epsilon)-Z_{0}(\bar{y}, \bar{z}, \epsilon)\right| \leqslant k(|y-\bar{y}|+|z-\bar{z}|) .
\end{gathered}
$$


Given these new functions $Y_{0}$ and $Z_{0}$, we may consider the system of differential equations

$$
\begin{aligned}
& \dot{y}=A(\epsilon) y+Y_{0}(y, z, \epsilon), \\
& \dot{z}=C(\epsilon) z+Z_{0}(y, z, \epsilon) .
\end{aligned}
$$

We will denote solutions of this system in the manner previously indicated for solutions of (1.3) except that in place of $x_{0}$ we will write $\left(y_{0}, z_{0}\right)$.

Our first theorem is as follows:

THEOREM 2.1. For each $\epsilon \in\left[0, \epsilon_{0}\right]$ there exists for (2.5) an integral manifold $M_{0}(\epsilon)$ represented by an equation of the form $z=F_{0}(y, \epsilon)$, where $F_{0}$ is a function defined and continuous on the domain $E^{2} \times\left[0, \epsilon_{0}\right]$. Moreover, $F_{0}$ satisfies the inequalities

$$
\begin{gathered}
\mid F_{0}(y, \epsilon) \leqslant \rho_{0}, \quad\left(y, \bar{y} \in E^{2}, 0 \leqslant \epsilon \leqslant \epsilon_{0}\right) \\
\left|F_{0}(y, \epsilon)-F_{0}(\bar{y}, \epsilon)_{i} \leqslant\right| y-\bar{y} \mid,
\end{gathered}
$$

and, also, $F_{0}(0, \epsilon)=0,0 \leqslant \epsilon \leqslant \epsilon_{0}$. Finally, for each $\epsilon \in\left[0, \epsilon_{0}\right], M_{0}(\epsilon)$ has the property that solutions of $(2.5)$ in $M_{0}(\epsilon)$ are defined for all $t$; i.e., if $x_{0} \in M_{0}(\epsilon)$ then $x\left(t, x_{0}, \epsilon\right)$ is defined for all $t \in E^{1}$.

Proof. Consider the integro-differential system

$$
\begin{aligned}
F_{0}\left(y_{0}, \epsilon\right) & =\int_{-\infty}^{0} e^{-C(\epsilon) \tau} Z_{0}\left(y(\tau), F_{0}(y(\tau), \epsilon), \epsilon\right) d \tau, \\
\dot{y}(t) & =A(\epsilon) y(t)+Y_{0}\left(y(t), F_{0}(y(t), \epsilon), \epsilon\right), \\
y(0) & =y_{0} \quad\left(y_{0} \in E^{2}, 0 \leqslant \epsilon \leqslant \epsilon_{0}\right) .
\end{aligned}
$$

We will show that in an appropriate function space there exists a unique function $F_{0}$ for which (2.6) holds. We will then show that for each $\epsilon \in\left[0, \epsilon_{0}\right]$ the equation $z=F_{0}(y, \epsilon)$ represents an integral manifold for (2.5) within which solutions of (2.5) are defined for all $t$ in $E^{1}$.

Let $S$ be the sct of functions $f: E^{2} \times\left[0, \epsilon_{0}\right]->E^{n-2}$ such that $f$ is continuous and such that

$$
\begin{aligned}
& f(0, \epsilon)=0, \\
& |f(y, \epsilon)| \leqslant \rho_{0}, \quad\left(y, \bar{y} \in E^{2}, 0 \leqslant \epsilon \leqslant \epsilon_{0}\right) . \\
& |f(y, \epsilon)-f(\bar{y}, \epsilon)| \leqslant|y-\bar{y}|
\end{aligned}
$$

Let $d$ be the metric on $S$ defined by

$$
\begin{gathered}
\left.d(f, g)=\sup \left\{f(y, \epsilon)-g(y, \epsilon) \mid: y \in E^{2}, 0 \leqslant \epsilon \leqslant \epsilon_{0}\right)\right\} \\
(f, g \in S),
\end{gathered}
$$

and note that, with respect to $d, S$ is a completc metric space. 
For each $f \in S$ we consider the differential equation

$$
\dot{y}=A(\epsilon) y+Y_{0}(y, f(y, \epsilon), \epsilon),
$$

solutions of which we denote by $y\left(t, y_{0}, f, \epsilon\right)$ with the understanding that $y\left(0, f, y_{0}, \epsilon\right)=y_{0}$. According to (2.3), $Y_{0}$ is uniformly bounded on its domain $D$; hence, any solution of (2.9) is defined for all $t$.

Now, define an operator $T$ on $S$ by setting

$$
\begin{gathered}
(T f)(y, \epsilon)=\int_{-\infty}^{0} e^{-C(\epsilon) \tau} Z_{0}(y(\tau, y, f, \epsilon), f(y(\tau, y, f, \epsilon), \epsilon), \epsilon) d \tau \\
\left(f \in S, y \in E^{2}, 0 \leqslant \epsilon \leqslant \epsilon_{0}\right) .
\end{gathered}
$$

The improper integral here converges by virtue of (2.1) and (2.5). Our intention is to show that $T$ is a contraction mapping of $S$ into itself.

It is a straightforward exercise to show that $T f$ as defined in $(2.10)$ is continuous on $E^{2} \times\left[0, \epsilon_{0}\right]$ and that $(T f)(0, \epsilon)=0,0 \leqslant \epsilon \leqslant \epsilon_{0}$. Also, using (2.1), (2.2), and (2.3), it is easy to see that

$$
:(T f)(y, \epsilon) \mid \leqslant K k \rho_{0} \int_{-\infty}^{0} e^{\alpha \tau} d \tau \leqslant \rho_{0} ;
$$

thus, $T f$ satisfies the boundedness condition required by (2.7). To prove that $T f$ satisfies the Lipschitz condition required by (2.7) and that $T$ is a contraction mapping, we reason as follows.

Let $(y, \epsilon),(\bar{y}, \epsilon) \in E^{2} \times\left[0, \epsilon_{0}\right]$ and let $f, g \in S$. Then, by (2.1),

$$
\begin{aligned}
& i(T f)(y, \epsilon)-(T g)(\bar{y}, \epsilon) \mid \\
& \quad \leqslant K \int_{-\infty}^{0} e^{\alpha \tau} \mid Z_{0}(y(\tau, y, f, \epsilon), f(y(\tau, y, f, \epsilon), \epsilon), \epsilon) \\
& \quad-Z_{0}(y(\tau, \bar{y}, g, \epsilon), g(y(\tau, \bar{y}, g, \epsilon), \epsilon) \mid d \tau .
\end{aligned}
$$

But, by (2.4); (2.7), and (2.8), there holds for all $\tau \in(-\infty, 0]$

$$
\begin{aligned}
& \left|Z_{0}(y(\tau, y, f, \epsilon), f(y(\tau, y, f, \epsilon), \epsilon), \epsilon)-Z_{0}(y(\tau, \bar{y}, g, \epsilon), g(y(\tau, \bar{y}, g, \epsilon), \epsilon), \epsilon)\right| \\
& \quad \leqslant\left|Z_{0}(y(\tau, y, f, \epsilon), f(y(\tau, y, f, \epsilon), \epsilon), \epsilon)-Z_{0}(y(\tau, \bar{y}, g, \epsilon), f(y(\tau, \bar{y}, g, \epsilon), \epsilon), \epsilon)\right| \\
& \quad+\left|Z_{0}(y(\tau, \bar{y}, g, \epsilon), f(y(\tau, \bar{y}, g, \epsilon), \epsilon), \epsilon)-Z_{0}(y(\tau, \bar{y}, g, \epsilon), g(y(\tau, \bar{y}, g, \epsilon), \epsilon), \epsilon)\right| \\
& \quad \leqslant 2 k|y(\tau, y, f, \epsilon)-y(\tau, \bar{y}, g, \epsilon)| \dashv k d(f, g) .
\end{aligned}
$$

Therefore,

$$
\begin{aligned}
& |(T f)(y, \epsilon)-(T g)(\bar{y}, \epsilon)| \\
& \quad \leqslant K k \alpha^{-1} d(f, g)+2 K k \int_{-\infty}^{0} e^{\alpha \tau}|y(\tau, y, f, \epsilon)-y(\tau, \bar{y} ; g, \epsilon)| d \tau .
\end{aligned}
$$


Now, by (2.9) and the variation of constants formula,

$$
\begin{aligned}
& y(\tau, y, f, \epsilon)=e^{A(\epsilon) \tau} y \\
& \quad+\int_{-\infty}^{0} e^{A(\epsilon)(\tau-s)} Y_{0}(y(s, y, f, \epsilon), f(y(s, y, f, \epsilon), \epsilon), \epsilon) d s
\end{aligned}
$$

also, this formula holds for $y(\tau, \bar{y}, g, \epsilon)$. Therefore, using (2.1) and estimates of the type leading to $(2.11)$, we find that

$$
\begin{aligned}
& y(\tau, y, f, \epsilon)-y(\tau, \bar{y}, g, \epsilon) \mid \\
& \leqslant K e^{-(\alpha / 2) \tau}\left(|y-\bar{y}|+2 k \alpha^{-1} d(f, g)\right) \\
& \quad+2 K k \int_{-\infty}^{0} e^{(\alpha / 2)(s-\tau)}|y(s, y, f, \epsilon)-y(\tau, \bar{y}, g, \epsilon)| d s \\
& \quad(-\infty<\tau<0) .
\end{aligned}
$$

Multiplying both sides of this equation by $e^{(\alpha / 2) \tau}$ and then applying reasoning of the type used to deduce Gronwall's Lemma (Lefschetz [9], p. 40), we obtain

$$
\begin{aligned}
& |y(\tau, y, f, \epsilon)-y(\tau, \bar{y}, g, \epsilon)| e^{(\alpha / 2) \tau} \\
& \leqslant K\left(|y-\bar{y}|+2 k \alpha^{-1} d(f, g)\right) e^{-2 \pi k \tau} \quad(-\infty<\tau \leqslant 0) .
\end{aligned}
$$

This and (2.2) allow us to write

$$
\begin{aligned}
& |y(\tau, y, f, \epsilon)-y(\tau, \bar{y}, g, \epsilon)| \\
& \leqslant K\left(|y-\bar{y}|+2 k \alpha^{-1} d(f, g)\right) e^{-(3 \alpha / 4) \tau} \quad(-\infty<\tau \leqslant 0) .
\end{aligned}
$$

Substitution of this into (2.11) and the use of (2.2) yield

$$
|(T f)(y, \epsilon)-(T g)(\bar{y}, \epsilon)| \leqslant|y-\bar{y}|+\frac{1}{2} d(f, g) .
$$

From this last inequality it easily follows that $T f$ satisfies the Lipschitz condition required by (2.7) and that $T$ is a contraction mapping.

Thus, $T$ is a contraction mapping of $S$ into itself and so, by the Banach Contraction Mapping Principle, $T$ must have a unique fixed point $F_{0} \in S$. $F_{0}$ is the required solution of (2.6).

Now, to show that for each $\epsilon, 0 \leqslant \epsilon \leqslant \epsilon_{0}$, the equation $z=F_{0}(y, \epsilon)$ represents an integral manifold for (2.5) within which solutions of (2.5) are defined for all $t$, we argue as follows. Let $\left(y_{0}, \epsilon\right) \in E^{2} \times[0, \epsilon]$ and let $z_{0}=F_{0}\left(y_{0}, \epsilon\right)$. Then set

$$
\bar{y}(t)=y\left(t, y_{0}, F_{0}, \epsilon\right), \bar{z}(t)-F_{0}(\bar{y}(t), \epsilon),
$$


and note that $\bar{y}(t)$ and $\bar{z}(t)$ are defined for all $t$. By (2.6)

$$
\bar{z}(t)=\int_{-x}^{0} e^{-C(\epsilon) \tau} Z_{0}\left(y\left(\tau, \bar{y}(t), F_{0}, \epsilon\right), F_{0}\left(y\left(\tau, \bar{y}(t), F_{0}, \epsilon\right), \epsilon\right), \epsilon\right) d \tau .
$$

However, since solutions of (2.9) satisfy the usual uniqueness conditions and since (2.9) is autonomous, $y\left(\tau, \bar{y}(t), F_{0}, \epsilon\right)=y\left(\tau+t, y_{0}, F_{0}, \epsilon\right)=\bar{y}(\tau+t)$. Substituting this into (2.13) and changing the variable of integration by letting $\tau+t \rightarrow \tau$, we obtain

$$
\bar{z}(t)=\int_{-\infty}^{t} e^{C(\epsilon)(t-\tau)} Z_{0}(\bar{y}(\tau), \bar{z}(\tau), \epsilon) d \tau
$$

From this result and the cquations

$$
\begin{gathered}
d \bar{y}(t) / d t=A(\epsilon) \bar{y}(t)+Y_{0}(\bar{y}(t), \bar{z}(t), \epsilon), \\
\bar{y}(0)=y_{0},
\end{gathered}
$$

which are a direct consequence of (2.12) and (2.6), it is easy to show that $\bar{y}(t)=y\left(t, y_{0}, z_{0}, \epsilon\right)$ and $\bar{z}(t)=z\left(t, y_{0}, z_{0}, \epsilon\right)$. Thus, $x\left(t, y_{0}, z_{0}, \epsilon\right)$ is defined for all $t$ and $z\left(t, y_{0}, z_{0}, \epsilon\right)=F_{0}\left(y\left(t, y_{0}, z_{0}, \epsilon\right), \epsilon\right)$.

Q.E.D.

Our next object is to obtain an attraction property of the manifold $M_{0}(\epsilon)$ with respect to solutions of (2.5) nearby it. To this end we introduce the following lemma.

\section{LeMma 2.2.1. On the domain}

$$
\begin{gathered}
R=\left\{(t, y, s, \epsilon): 0 \leqslant t<\infty, 0 \leqslant|y|<\infty, 0 \leqslant|z| \leqslant r_{1}, 0 \leqslant \epsilon \leqslant \epsilon_{0}\right\} \\
\left(r_{1}=(K+1)^{-1} \rho_{0}\right)
\end{gathered}
$$

there is defined a continuous function $h$ with range in $E^{n-2}$ such that if $x\left(t, y_{0}, z_{0}, \epsilon\right)$ is a solution of (2.5) with $\left|z_{0}\right| \leqslant r_{1}$ then $x\left(t, y_{0}, z_{0}, \epsilon\right)$ is defined for all $t>0$ and satisfies the identity

$$
\begin{array}{r}
z\left(t, y_{0}, z_{0}, \epsilon\right)=h\left(t, y\left(t, y_{0}, z_{0}, \epsilon\right), z_{0}, \epsilon\right) \\
(0 \leqslant t<\infty) .
\end{array}
$$

Moreover, $h$ satisfies the inequalities

$$
\begin{gathered}
i h(t, y, z, \epsilon) \mid \leqslant r_{1}, . \\
|h(t, y, z, \epsilon)-h(t, \bar{y}, \bar{z}, \epsilon)| \leqslant\left|y-\bar{y}+2 K e^{-(\alpha / 4) t}\right| z-\bar{z} \mid \\
((t, y, z, \epsilon),(t, \bar{y}, \bar{z}, \epsilon) \in R) .
\end{gathered}
$$


Proof. Consider the integro-differential system

$$
\begin{aligned}
h\left(t_{0}, y_{0}, z_{0}, \epsilon\right) & =e^{C(\epsilon) t_{0} z_{0}}+\int_{0}^{t} e^{C(\epsilon)\left(t_{0}-\tau\right)} Z_{0}\left(y(\tau), h\left(\tau, y(\tau), z_{0}, \epsilon\right), \epsilon\right) d \tau, \\
\dot{y}(t) & =A(\epsilon) y(t)+Y_{0}\left(y(t), h\left(t, y(t), z_{0}, \epsilon\right), \epsilon\right), t \geqslant 0, \\
y\left(t_{0}\right) & =y_{0}, \quad\left(\left(t_{0}, y_{0}, z_{0}, \epsilon\right) \in R\right) .
\end{aligned}
$$

By arguments paralleling those used in the proof of Theorem 2.1, one can show that there exists a unique continuous function $h: R \rightarrow E^{n-2}$ for which (2.16) and (2.17) hold. Following this it is easy to show that $h$ has the property embodied in (2.15). For lack of space we omit the details.

TheOREM 2.2. Let $F_{0}$ be as in Theorem 2.1 and let $r_{1}$ be as in (2.14). If $x\left(t, y_{0}, z_{0}, \epsilon\right)$ is a solution of (2.7) for which $\left|z_{0}\right| \leqslant r_{1}$, then $x\left(t, y_{0}, z_{0}, \epsilon\right)$ is defined for all $t \geqslant 0$ and its components satisfy the inequality

$$
\begin{array}{r}
\mid z\left(t, y_{0}, z_{0}, \epsilon\right)-F_{0}\left(y\left(t, y_{0}, z_{0}, \epsilon\right), \epsilon\right) \\
\leqslant 4 K r_{1} e^{-(\alpha / 4) t_{1}} \quad(0 \leqslant t<\infty) .
\end{array}
$$

- Proof. First, we show that $F_{0}$ on its domain $E^{2} \times\left[0, \epsilon_{0}\right]$ is uniformly bounded by $r_{1}$. This follows from the fixed point property of $F_{0}$ exhibited in the proof of Theorem 2.1 and from (2.1), (2.2), and (2.3); specifically, for all $(y, \epsilon) \in E^{2} \times\left[0, \epsilon_{0}\right]$ we have

$$
{ }_{i} F_{0}(y, \epsilon)|=|\left(T F_{0}\right)(y, \epsilon) \mid \leqslant 2 K k \rho_{0} \int_{-\infty}^{0} e^{\alpha \tau} d \tau \leqslant r_{1} .
$$

This inequality and Theorem 2.2 imply that all solutions of (2.5) lying in $M_{0}(\epsilon)$ are subject to (2.15).

Now, let $x\left(t, y_{0}, z_{0}, \epsilon\right)$ be any solution of (2.5) for which $\left|z_{0}\right| \leqslant r_{1}$. By Theorem 2.2 this solution is defined for all $t \geqslant 0$. Choose any $t_{1} \geqslant 0$ and then let $y=y\left(t_{1}, y_{0}, z_{0}, \epsilon\right)$ and $z=F_{0}(y, \epsilon)$. For $y$ and $z$ so defined, consider the solution $x(t, y, z, \epsilon)$, which by Theorem 2.1 lies in $M_{0}(\epsilon)$ and is defined for all $t$. We set $y_{1}=y\left(-t_{1}, y, z, \epsilon\right)$ and $z_{1}=z\left(-t_{1}, y, z, \epsilon\right)$ and then note that $y=y\left(t_{1}, y_{1}, z_{1}, \epsilon\right)$ and $z=z\left(t_{1}, y_{1}, z_{1}, \epsilon\right)$. But on the other hand, by (2.15),

$$
\begin{aligned}
& z\left(t_{1}, y_{0}, z_{0}, \epsilon\right)=h\left(t_{1}, y\left(t_{1}, y_{0}, z_{0}, \epsilon\right), z_{0}, \epsilon\right)=h\left(t_{1}, y, z_{0}, \epsilon\right), \\
& z\left(t_{1}, y_{1}, z_{1}, \epsilon\right)-h\left(t_{1}, y\left(t_{1}, y_{1}, z_{1}, \epsilon\right), z_{1}, \epsilon\right)=h\left(t_{1}, y, z_{1}, \epsilon\right) .
\end{aligned}
$$

Hence, by (2.16)

$$
\left|z\left(t_{1}, y_{0}, z_{0}, \epsilon\right)-z\left(t_{1}, y_{1}, z_{1}, \epsilon\right) \leqslant 2 K e^{-(\alpha / 4) t_{1}}\right| z_{0} \quad z_{1} \cdot .
$$


Since, however, $x\left(t, y_{1}, z_{1}, \epsilon\right)$ is a solution of $(2.5)$ lying in $M_{0}(\epsilon)$, it follows that

$$
z\left(t_{1}, y_{1}, z_{1}, \epsilon\right)=F_{0}\left(y\left(t_{1}, y_{1}, z_{1}, \epsilon\right), \epsilon\right)=F(y, \epsilon)
$$

and, also, that $\left|z_{1}\right| \leqslant r_{1}$. Recalling, finally, that $\left|z_{0}\right| \leqslant r_{1}$ and that $y=y\left(t_{1}, y_{0}, z_{0}, \epsilon\right)$, we obtain (2.19) with $t$ replaced by $t_{1}$. Since $t_{1} \geqslant 0$ was chosen arbitrarily, the proof is complete.

CoRollary 2.2.1. Let $M_{0}(\epsilon)$ be as in Theorem 2.1 and let $r_{1}$ be as in (2.14). Suppose that $x\left(t, y_{0}, z_{0}, \epsilon\right)$ is a solution of $(2.5)$ defined for all $t \leqslant 0$ and having the property that $\mid z\left(t, y_{0}, z_{0}, \epsilon\right)_{\mid} \leqslant r_{1}$ for all $t \leqslant 0$. Then $x\left(t, y_{0}, z_{0}, \epsilon\right)$ lies in $M_{0}(\epsilon)$.

The proof of this corollary is easy enough so that we need not render it here.

From the manner in which $Y_{0}$ and $Z_{0}$ were constructed and from the inequality $r_{1}<\rho_{0}$, it follows that Eqs. (1.3) and (2.5) agree on the domain $B^{n}\left(r_{1}\right) \times\left[0, \epsilon_{0}\right]$. Therefore, by Theorems 2.1 and 2.2 and Corollary 2.2.1 we have the following results.

TheOREM 2.3. Let $r_{0}$ and $\epsilon_{0}$ be as in $\left(\mathrm{H}_{1}\right)$. Then, for each $\epsilon \in\left[0, \epsilon_{0}\right]$ there exists for (1.3) a local integral manifold $M^{2}(\epsilon)$ represented by an equation of the form $z=F(y, \epsilon)$, where $F$ is a function defined and continuous on a domain $B^{2}\left(r_{1}\right) \times\left[0, \epsilon_{0}\right], r_{1} \in\left(0, r_{0}\right]$, and satisfying there the conditions

$$
\begin{aligned}
& F(0, \epsilon)=0, \\
& |F(y, \epsilon)-F(\bar{y}, \epsilon)| \leqslant|y-\bar{y}| . \quad\left(y, \bar{y} \in B^{2}\left(r_{1}\right), 0 \leqslant \epsilon \leqslant \epsilon_{0}\right) .
\end{aligned}
$$

Moreover, $r_{1}$ can be chosen so that $M^{2}(\epsilon)$ has the following properties.

(i) There exist constants $L>0$ and $\beta>0$ such that if $x\left(t, x_{0}, \epsilon\right)$ is any solution of (1.3) with $\left|x_{0}\right| \leqslant r_{1}$ then for all $t>0$ such that $\left|x\left(\tau, x_{0}, \epsilon\right)\right| \leqslant r_{1}$, $0 \leqslant \tau \leqslant t$, there holds the inequality

$$
\left|z\left(t, x_{0}, \epsilon\right)-F\left(y\left(t, x_{0}, \epsilon\right), \epsilon\right)\right| \leqslant L e^{-\beta t} .
$$

(ii) If $x\left(t, x_{0}, \epsilon\right)$ is a solution of (1.3) for which $\left|x\left(t, x_{0}, \epsilon\right)\right| \leqslant r_{1}, t \leqslant 0$, then $x\left(t, x_{0}, \epsilon\right) \in M^{2}(\epsilon), t \leqslant 0$.

Proof. See the remark preceding this theorem. For $r_{1}$ here we take $r_{1}$ as in (2.14), and for $F$ we take the restriction of $F_{0}$ in Theorem 2.1 to $B^{2}\left(r_{1}\right) \times\left[0, \epsilon_{0}\right]$. For $L$ and $\beta$ we take $4 K r_{1}$ and $\alpha / 4$, where $K$ and $\alpha$ arc as in $\left(\mathrm{H}_{1}\right)$.

The following two corollaries, whose proofs are trivial, will be of use to us in the next section. 
Corollary 2.3.1. For each $\epsilon \in\left[0, \epsilon_{0}\right], M^{2}(\epsilon)$ is homeomorphic to the open disk $B^{2}\left(r_{1}\right)$, a homeomorphism being given by the correspondence $y \leftrightarrow(y, F(y, \epsilon))$, $y \in B^{2}\left(r_{1}\right)$. Also, for each $\epsilon \in\left[0, \epsilon_{0}\right]$ the origin $x=0$ is contained in $M^{2}(\epsilon)$.

CoROLlaRY 2.3.2. For $F$ as in Theorem 2.3, we consider the differential equation

$$
y=A(\epsilon) y+Y(y, F(y, \epsilon), \epsilon),
$$

solutions of which we denote by $y\left(t, y_{0}, \epsilon\right)$ with the understanding that $y\left(0, y_{0}, \epsilon\right)=y_{0}$. If $x\left(t, x_{0}, \epsilon\right)$ is any solution of (1.3) for which $x_{0} \in M^{2}(\epsilon)$, i.e., for which $x_{0}$ has the form $x_{0}=\left(y_{0}, F\left(y_{0}, \epsilon\right)\right), y_{0} \in B^{2}\left(r_{1}\right)$, then the components of $x\left(t, x_{0}, \epsilon\right)$ are related to the solution $y\left(t, y_{0}, \epsilon\right)$ of $(2.20)$ through the equations

$$
y\left(t, x_{0}, \epsilon\right)=y\left(t, y_{0}, \epsilon\right), z\left(t, x_{0}, \epsilon\right)=F\left(y\left(t, y_{0}, \epsilon\right), \epsilon\right),
$$

these equations holding at all $t$ for which $x\left(t, x_{0}, \epsilon\right)$ remains in $B^{n}\left(r_{1}\right)$.

\section{The Existence of the Closed Orbits $\gamma_{1}(\epsilon)$ AND $\gamma_{2}(\epsilon)$}

In this section we will study the behavior for $\epsilon>0$ of solutions of (1.3) in a neighborhood of the origin $x=0$. Our first theorem is as follows.

Theorem 3.1. Let $r_{0}$ and $\epsilon_{0}$ be as in $\left(\mathrm{H}_{1}\right)$. Then, for each $\epsilon \in\left(0, \epsilon_{0}\right]$ there exists for (1.3) a local integral manifold $M^{n-2}(\epsilon)$ homeomorphic to an open ball in $E^{n-2}$, containing the origin $x=0$, and having the property that if $x_{0} \in M^{n-2}(\epsilon)$ then $x\left(t, x_{0}, \epsilon\right) \rightarrow 0$ as $t \rightarrow+\infty$. Moreover, there exists a positive-valued continuous function $\sigma=\sigma(\epsilon), 0<\epsilon \leqslant \epsilon_{0}$, approaching zero as $\epsilon \rightarrow 0+$, for which the following assertions are true.

(i) For any $\epsilon \in\left(0, \epsilon_{0}\right]$ a solution $x\left(t, x_{0}, \epsilon\right)$ of $(1.3)$ with $x_{0} \in B^{n}(\sigma(\epsilon))$ $-M^{n-2}(\epsilon)$ can not remain in $B^{n}(\sigma(\epsilon))$ for all $t>0$.

(ii) For each $\epsilon \in\left(0, \epsilon_{0}\right]$ the manifold $M^{n-2}(\epsilon)$ con be represented by an equation of the form $y=G(z, \epsilon)$, where $G$ is a function defined and continuous on the domain

$$
\left\{(z, \epsilon): 0 \leqslant|z|<\sigma(\epsilon), 0<\epsilon \leqslant \epsilon_{0}\right\} .
$$

The proof is a slight revision of that of Theorem 4.1 on p. 330 of [4], the modifications being made to take into account the presence of the parameter $\epsilon$ in (1.3) and to incorporate the remarks made in lines 10-14 on p. 342 of [4].

We consider now for $\epsilon>0$ those solutions of (1.3) in a neighborhood of the origin which do not approach the origin as $t \rightarrow+\infty$. For these solutions we have the following two theorems. 
THEOREM 3.2. Let $\epsilon_{0}$ be as in $\left(\mathrm{H}_{1}\right)$ and let $r_{1}$ and $M^{2}(\epsilon)$ be as in Theorem 2.3 . Then, there exist numbers $r_{2} \in\left(0, r_{1}\right]$ and $\epsilon_{0}^{\prime} \in\left(0, \epsilon_{0}\right]$ such that if $x\left(t, x_{0}, \epsilon\right)$ is a solution of (1.3) with $\left|x_{0}\right|<r_{2}$ and $0<\epsilon \leqslant \epsilon_{0}^{\prime}$ then $\left|x\left(t, x_{0}, \epsilon\right)\right|<r_{1}$, $t>0$. Also, for each $\epsilon \in\left(0, \epsilon_{0}^{\prime}\right]$ there exists an $r(\epsilon) \in\left(0, r_{2}\right]$ so that if $x\left(t, x_{0}, \epsilon\right)$ is a solution of the type just described, then $x\left(t, x_{0}, \epsilon\right)$ has a nonempty positivelimiting set $\omega^{+}\left([8]\right.$, pp. 57-58) contained in $M^{2}(\epsilon) \cap B^{n}(r(\epsilon))$. Moreover, $\omega^{+}$ is compact, connected, and invariant, and $x\left(t, x_{0}, \epsilon\right) \rightarrow \omega^{+}$as $t \rightarrow+\infty$. Finally, $r(\epsilon)$ can be chosen so that $r(\epsilon) \rightarrow 0$ as $\epsilon \rightarrow 0+$.

Proof. In order to simplify our notation, we will throughout this proof use Eq. (1.1) in place of Eq. (1.3).

Let $r_{0}$ be as in $\left(\mathrm{H}_{1}\right)$. From hypothesis $\left(\mathrm{H}_{5}\right)$ and the autonomous property of (1.1) it follows that for $\epsilon=0$ the origin $x=0$ is a uniformly asymptotically stable equilibrium point of (1.1) ([5], p. 63). Therefore, by well-known theorems ([5], pp. 64 and 70), there exists a $\rho_{1} \in\left(0, r_{0}\right]$ such that on the domain $B^{n}\left(\rho_{1}\right)$ there is defined a continuously differentiable Liapunov function $V$ such that (i) $V$ is positive-definite on $B^{n}\left(\rho_{1}\right)$ (i.e., $V(0)=0$ and $V(x)>0$ for $\left.0<|\boldsymbol{x}|<\rho_{1}\right)$, and (ii) the function $\dot{V}$ defined by

$$
\dot{V}(x)=(\operatorname{grad} V(x)) \cdot(P(0) x+X(x, 0)) \quad\left(x \in B^{n}\left(p_{1}\right)\right)
$$

is negative-definite on $B^{n}\left(\rho_{1}\right)$ (i.e., $-\dot{V}$ is positive-definite). We immediately extend the domain of definition of $\dot{V}$ by letting

$$
\begin{gathered}
\dot{V}(x, \epsilon)=(\operatorname{grad} V(x)) \cdot(P(\epsilon) x+X(x, \epsilon)) \\
\left(x \in B^{n}\left(\rho_{1}\right), 0 \leqslant \epsilon \leqslant \epsilon_{0}\right) .
\end{gathered}
$$

Without loss of generality we may assume that $\rho_{1} / 2<r_{1}$.

Arguing from the continuity of $V$ and its positive-definite property, one can show the existence of numbers $m>0$ and $r_{2}, 0<r_{2}<\rho_{1} / 2<r_{1}$, such that

$$
\begin{aligned}
& V(x) \geqslant m \quad\left(|x|=\rho_{1} / 2\right), \\
& V(x) \leqslant m / 2 \quad\left(|x| \leqslant r_{2}\right) .
\end{aligned}
$$

Since $\dot{V}$ is continuous on $B^{n}\left(\rho_{1}\right) \times\left[0, \epsilon_{0}\right]$ and, as a function of $x$, is negativedefinite when $\epsilon=0$, it is possible to choose an $\epsilon_{0}^{\prime} \in\left(0, \epsilon_{0}\right]$ so that $\dot{V}$ is negativevalued on the domain $\left(B^{n}\left(\rho_{1}\right)-B^{n}\left(r_{2}\right)\right) \times\left[0, \epsilon_{0}^{\prime}\right]$. Having selected such an $\epsilon_{0}^{\prime}$, it is then possible to choose for each $\epsilon \in\left(0, \epsilon_{0}^{\prime}\right]$ an $r(\epsilon) \in\left(0, r_{2}\right]$ in such a way that $r(\epsilon) \rightarrow 0$ as $\epsilon \rightarrow 0+$ and

$$
V(x, \epsilon)<0 \quad\left(x \in B^{n}\left(\rho_{1}\right)-B^{n}(r(\epsilon)), \quad 0<\epsilon \leqslant \epsilon_{0}^{\prime}\right) .
$$

Now, using (3.1), (3.2), and (3.3) it is easy to show that if $x\left(t, x_{0}, \epsilon\right)$ is a solution of (1.1) for which $\left|x_{0}\right|<r_{2}$ and $0<\epsilon \leqslant \epsilon_{0}^{\prime}$ then $\left|x\left(t, x_{0}, \epsilon\right)\right|<r_{1}$, 
$t>0$, and $\left|x\left(t, x_{0}, \epsilon\right)\right|<r(\epsilon)$ for $t$ greater than some $t_{0}>0$. Since the closure of $B^{n}(r(\epsilon))$ is compact, it follows that $x\left(t, x_{0}, \epsilon\right)$ must have a nonempty compact, connected, and invariant positive-limiting set $\omega^{+}$in $B^{n}(r(\epsilon))$ and that $x\left(t, x_{0}, \epsilon\right) \rightarrow \omega^{+}$as $t \rightarrow+\infty$ ([8], p. 58). From Assertion (i) of Theorem 2.3 it follows that $\omega^{+} \subset M^{2}(\epsilon)$.

Q.E.D.

TheOREM 3.3. Let $r_{2}, \epsilon_{0}^{\prime}$, and $r(\epsilon)$ be as in Theorem 3.2. Then, for each $\epsilon \in\left(0, \epsilon_{0}^{\prime}\right]$ there exists a $\rho(\epsilon) \in(0, r(\epsilon)]$ such that if $x\left(t, x_{0}, \epsilon\right)$ is a solution of (1.3) for which $\left|x_{0}\right|<r_{2}$ and if $x\left(t, x_{0}, \epsilon\right) \nrightarrow 0$ as $t \rightarrow+\infty$ (see Theorem 3.1), then $x\left(t, x_{0}, \epsilon\right)$ has its positive-limiting set (whose existence is guaranteed by Theorem 3.2) in $M^{2}(\epsilon) \cap B^{n}(r(\epsilon))-B^{n}(\rho(\epsilon))$.

Proof. Choose any $\epsilon \in\left(0, \epsilon_{0}^{\prime}\right]$. Throughout this proof $\epsilon$ will be fixed and, where appropriate, suppressed in our notation.

By $\left(\mathrm{H}_{4}\right)$ the eigenvalues of $A(\epsilon)$ have their real parts positive. Hence, by a theorem of Liapunov ([9], pp. 356-358) there exists a real $2 \times 2$ symmetric matrix $Q=Q(\epsilon)$ such that the quadratic form $W=W(y)=y^{*} Q y\left({ }^{*}\right.$ denotes transpose) is positive-definite and such that for all $y \in E^{2}$

$$
y^{*}\left(A^{*}(\epsilon) Q+Q A(\epsilon)\right) y=|y|^{2} .
$$

Let $r_{0}$ be as in $\left(\mathrm{H}_{1}\right)$. Regarding $W$ as a Liapunov function for (1.3), we see that on the basis of (3.4)

$$
\begin{gathered}
W(x)=\mid y_{1}^{2}+Y^{*}(x, \epsilon) Q y+y^{*} Q Y(x, \epsilon) \\
\left(x=(y, z), x \in B^{n}\left(r_{0}\right)\right) .
\end{gathered}
$$

Hence, by $\left(\mathrm{H}_{2}\right)$ and $\left(\mathrm{H}_{3}\right)$ there exists an $\eta=\eta(\epsilon), 0<\eta(\epsilon)<r(\epsilon)$, such that on the domain

$$
R=\{x=(y, z): 0 \leqslant|z| \leqslant 3|y|, 0 \leqslant ! y \mid \leqslant \eta\}
$$

$W$ is positive-definite, i.e., $W(0)=0$ and $W(x)>0$ for $x \neq 0$ in $R$.

From the continuity of $W$ and its positive-definite property there follow the existence of numbers $m>0$ and $\rho=\rho(\epsilon), 0<\rho(\epsilon)<\eta(\epsilon)$, such that

$$
\begin{aligned}
& W(y) \geqslant m \quad(|y|=\eta), \\
& W(y) \leqslant \frac{m}{2} \quad(0 \leqslant|y| \leqslant \rho) .
\end{aligned}
$$

We will prove our theorem for $\rho=\rho(\epsilon)$ as just chosen.

Let $x\left(t, x_{0}, \epsilon\right)$ be a solution of (1.3) such that $\left|x_{0}\right|<r_{2}$ and such that $x\left(t, x_{0}, \epsilon\right) \nrightarrow 0$ as $t \rightarrow+\infty$. Then, by Theorem $3.2 x\left(t, x_{0}, \epsilon\right)$ has a nonempty connected positive-limiting set $\omega^{+} \subset M^{2}(\epsilon) \cap B^{n}(r(\epsilon))$. If $\omega^{+} \cap B^{n}(\rho(\epsilon))$ is empty then there is nothing to prove. Hence, let us suppose that there exists 
an $x_{1} \in \omega^{+} \cap B^{n}(\rho(\epsilon))$. Since $x\left(t, x_{0}, \epsilon\right) \nrightarrow 0$ as $t \rightarrow+\infty$ and since $\omega^{+}$is connected, we may assume that $x_{1} \neq 0$. We will deduce a contradiction.

Let $\delta_{1}=\mid x_{1} / 2$. Then, since $W$ is continuous and positive-definite, there exist numbers $\mu>0$ and $\delta_{2}, 0<\delta_{2}<\delta_{1}$, such that

$$
\begin{gathered}
W(y) \geqslant \mu \quad\left(|y|=\delta_{1}\right), \\
W(y) \leqslant \frac{\mu}{2} \quad\left(0 \leqslant|y| \leqslant \delta_{2}\right) .
\end{gathered}
$$

Now, by assertion (ii) of Theorem 3.2 there exists a $t_{0}>0$ such that

$$
\left|z\left(t, x_{0}, \epsilon\right)-F\left(y\left(t, x_{0}, \epsilon\right), \epsilon\right)\right| \leqslant \delta_{2}, \quad t>t_{0} .
$$

This and (2.19) imply that

$$
\left|z\left(t, x_{0}, \epsilon\right)\right| \leqslant \delta_{2}+\left|y\left(t, x_{0}, \epsilon\right)\right|, \quad t>t_{0}
$$

Moreover, recalling that $x_{1} \in \omega^{+} \cap B^{n}(r(\epsilon))$ and that $\delta_{1}<\left|x_{1}\right|$, we may assume that $t_{0}$ is large enough so that

$$
\begin{aligned}
\left|y\left(t_{0}, x_{0}, \epsilon\right)\right| & >\delta_{1}, \\
\left|y\left(t, x_{0}, \epsilon\right)\right| & <r(\epsilon) \quad\left(t>t_{0}\right) .
\end{aligned}
$$

Now suppose that there exists a $t_{1}>t_{0}$ such that $\left|y\left(t_{1}, x_{0}, \epsilon\right)\right|<\delta_{2}$. Then, from standard connectedness arguments there follows the existence of numbers $t_{2}$ and $t_{3}, t_{0}<t_{2}<t_{3}<t_{1}$, such that

$$
\begin{aligned}
\left|y\left(t_{2}, x_{0}, \epsilon\right)\right|= & \delta_{1}, \\
\delta_{2} \leqslant\left|y\left(t, x_{0}, \epsilon\right)\right| \leqslant \delta_{1} & \left(t_{2} \leqslant t \leqslant t_{3}\right), \\
\left|y\left(t_{3}, x_{0}, \epsilon\right)\right|= & \delta_{2} .
\end{aligned}
$$

But, taking into account (3.7), we see that $\left|z\left(t, x_{0}, \epsilon\right)\right| \leqslant 2\left|y\left(t, x_{0}, \epsilon\right)\right|$ when $t_{2} \leqslant t \leqslant t_{3}$. Thus, $x\left(t, x_{0}, \epsilon\right) \in R$ when $t_{2} \leqslant t \leqslant t_{3}$. However, $W$ is positive-definite on $R$; hence, by (3.6) and (3.9) we have a contradiction. Therefore, $t_{1}$ can not exist, which means that $\mid y\left(t, x_{0}, \epsilon\right) \geqslant \delta_{2}$ for all $t>t_{0}$.

This conclusion and (3.7) and (3.8) imply that $x\left(t, x_{0}, \epsilon\right)$ remains in the region $\left\{x=(y, z): 0 \leqslant|z| \leqslant 2|y|, \delta_{2} \leqslant|y|<r(\epsilon)\right\}$ for all $t>t_{0}$. However, recalling that $\delta_{2}<\delta_{1}<\rho(\epsilon)<\eta(\epsilon)<r(\epsilon)$, we note that $W$ is positive-valued in the sub-region $\{x=(y, z): 0 \leqslant|z| \leqslant 2|y|$, $\delta_{\mathbf{2}} \leqslant|y| \leqslant \eta(\epsilon)$. From this observation, (3.5), and the known inequalities $\delta_{2}<\left|x_{1}\right|<\rho(\epsilon)$, it follows that $x_{1}$ can not be in the positive-limiting set of $x\left(t, x_{0}, \epsilon\right)$, which is a contradiction.

Q.E.D.

COROLLARY 3.3.1. Let $r_{2}$ and $\epsilon_{0}^{\prime}$ be as in Theorem 3.2. If for any $\epsilon \in\left(0, \epsilon_{0}^{\prime}\right]$ 
$x\left(t, x_{0}, \epsilon\right)$ is a nonzero solution of (1.3) for which $x_{0} \in M^{2}(\epsilon) \cap B^{n}\left(r_{2}\right)$ then $x\left(t, x_{0}, \epsilon\right) \nrightarrow 0$ as $t \rightarrow+\infty$.

Proof. Fix any $\epsilon \in\left(0, \epsilon_{0}^{\prime}\right]$ and let $\eta=\eta(\epsilon), R$, and $W$ be as in the proof of Theorem 3.3. From (2.19) it easily follows that $M^{2}(\epsilon) \cap B^{n}(\eta)$ is contained in $R$. Now let $x\left(t, x_{0}, \epsilon\right)$ be a solution of (1.3) with $x_{0} \in M^{2}(\epsilon) \cap B^{n}\left(r_{2}\right)$ and $x_{0} \neq 0$. By Theorem $3.2\left|x\left(t, x_{0}, \epsilon\right)\right|<r_{1}$ for all $t>0$; hence, $x\left(t, x_{0}, \epsilon\right) \in M^{2}(\epsilon)$ for all $t>0$. Since $W$ and $W$ are both positive-definitc on $R$ it follows that $x\left(t, x_{0}, \epsilon\right) \rightarrow 0$ as $t \rightarrow+\infty$.

Q.E.D.

The next theorem, for which we need a trivial lemma, will give us information concerning the structure of the positive-limiting sets whose existence we deduced in Theorem 3.2 .

LeMMA 3.4.1. Let $r_{2}$ and $\epsilon_{0}^{\prime}$ be as in Theorem 3.2. Then, there exists an $r_{3} \in\left(0, r_{2}\right]$ such that for each $\in \in\left[0, \epsilon_{0}\right]$ the origin $x=0$ is the only equilibrium point of (1.3) inside $B^{n}\left(r_{3}\right)$.

Proof. From $\left(\mathrm{H}_{1}\right)$ it follows that the matrices $A$ and $C$ in (1.3) are continuous in $\epsilon$. From $\left(\mathrm{H}_{4}\right)$ it follows that each of these matrices has nonzero eigenvalues for all $\epsilon \in\left[0, \epsilon_{0}\right]$. Hence, there exists a constant $\eta>0$ such that

$$
\begin{aligned}
& |A(\epsilon) y| \geqslant \eta|y|, \\
& |C(\epsilon) z| \geqslant \eta|z| .
\end{aligned} \quad\left(y \in E^{2}, z \in E^{n-2}, 0 \leqslant \epsilon \leqslant \epsilon_{0}\right) .
$$

From $\left(\mathrm{H}_{2}\right)$ and $\left(\mathrm{H}_{3}\right)$ it follows that there exists a constant $r_{3} \in\left(0, r_{2}\right]$ such that

$$
\begin{aligned}
& |Y(x, \epsilon)| \leqslant(\eta / 2)|x|, \\
& |Z(x, \epsilon)| \leqslant(\eta / 2)|x| .
\end{aligned}
$$

The above inequalities imply the result required by our lemma.

Theorem 3.4. For $r_{2}$ and $\epsilon_{0}^{\prime}$ as in Theorem 3.2, there exists an $\epsilon_{1} \in\left(0, \epsilon_{0}^{\prime}\right]$ such that the following is true. Let $x\left(t, x_{0}, \epsilon\right)$ be a solution of (1.3) for which $\left|x_{0}\right|<r_{2}$ and $0<\epsilon<\epsilon_{1}$, and suppose that $x\left(t, x_{0}, \epsilon\right) \nrightarrow 0$ as $t \rightarrow+\infty$. Let $x_{1}$ be any point in the positive-limiting set of $x\left(t, x_{0}, \epsilon\right)$. Then, $x\left(t, x_{1}, \epsilon\right)$ is defined for all $t,-\infty<t<\infty$, and, for such $t$, is contained in the set $M^{2}(\epsilon) \cap\left[B^{n}(r(\epsilon))-B^{n}(\rho(\epsilon))\right]$, where $r(\epsilon)$ and $\rho(\epsilon)$ are as in Theorems 3.2 and 3.3 respectively. Moreover, $x\left(t, x_{1}, \epsilon\right)$ satisfies one and only one of the following descriptions:

(i) $x\left(t, x_{1}, \epsilon\right)$ is a closed orbit of (1.3), which, regarded as a closed Jordan curve in $M^{2}(\epsilon)$, contains the origin $x=0$ in its interior. 
(ii) There exist two distinct closed orbits $\Gamma^{+}$and $\Gamma^{-}$of (1.3) such that $x\left(t, x_{1}, \epsilon\right) \rightarrow \Gamma^{+}$as $t \rightarrow+\infty$ and $x\left(t, x_{1}, \epsilon\right) \rightarrow \Gamma^{-}$as $t \rightarrow-\infty$. These orbits lie in $M^{2}(\epsilon)$ and are concentric about the origin $x=0$.

Finally, if $x_{0} \in M^{2}(\epsilon)$ then $x\left(t, x_{1}, \epsilon\right)$ must satisfy (i).

Proof. For $r_{3}$ as in Lemma 3.4.1 we choose $\epsilon_{1} \in\left(0, \epsilon_{0}\right]$ so that $r(\epsilon)<r_{3}$ for all $\epsilon \in\left(0, \epsilon_{1}\right]$. Such a choice is possible because $r(\epsilon) \rightarrow 0$ as $\epsilon \rightarrow 0+$. Now let $x\left(t, x_{0}, \epsilon\right)$ be a solution of (1.3) for which $\left|x_{0}\right|<r_{2}$ and $0<\epsilon<\epsilon_{1}$, and suppose that $x\left(t, x_{0}, \epsilon\right) \nrightarrow 0$ as $t \rightarrow+\infty$. Let $x_{1}$ be any point in the positive-limiting set $\omega^{+}$of $x\left(t, x_{0}, \epsilon\right)$. From the invariance of $\omega^{+}$ (Theorem 3.2) and from Theorem 3.3 it follows that $x\left(t, x_{1}, \epsilon\right)$ is contained in $M^{2}(\epsilon) \cap\left[B^{n}(r(\epsilon))-B^{n}(\rho(\epsilon))\right]$ and, hence, is defined for all $t \in E^{\mathbf{1}}$.

Now, referring to Corollary 2.3.2, we let $y\left(t, y_{1}, \epsilon\right)$ be the solution of (2.20) corresponding through (2.21) to $x\left(t, x_{1}, \epsilon\right)$. Then, by (2.19) and (2.21), $y\left(t, y_{1}, \epsilon\right)$ remains in $\left(B^{2}(r(\epsilon))-B^{2}(\rho(\epsilon) / 2)\right)$ for all $t \in E^{1}$. By the above choice of $\epsilon_{1}$ and by Corollary 2.3.2, this region contains no equilibrium points of (2.20). These observations together with the autonomous property of (2.20) and a theorem of Bendixson ([9], p. 230) imply the following: either $y\left(t, y_{1}, \epsilon\right)$ is a closed orbit of (2.20) or (2.20) has two closed orbits $\Lambda^{-}$and $\Lambda^{-}$ such that $y\left(t, y_{1}, \epsilon\right) \rightarrow \Lambda^{+}$as $t \rightarrow+\infty$ and $y\left(t, y_{1}, \epsilon\right) \rightarrow \Lambda^{-}$as $t \rightarrow-\infty$. In the latter case a simple argument shows that $\Lambda^{+}$and $\Lambda^{-}$are distinct. In both cases a theorem of Poincare ([9], p. 200) implies that the orbits in question contain the origin $y=0$ in their interiors. Assertions (i) and (ii) of our theorem now trivially follow.

To prove the last assertion made in our theorem, suppose $x_{0} \in M^{2}(\epsilon)$. Then, again applying Corollary 2.3.2, we let $y\left(t, y_{0}, \epsilon\right)$ be the solution of (2.20) corresponding to $x\left(t, x_{0}, \epsilon\right)$. Since $y\left(t, y_{1}, \epsilon\right)$ is the positive-limiting set of $y\left(t, y_{0}, \epsilon\right)$, it follows from Bendixson's Theorem cited above that $y\left(t, y_{1}, \epsilon\right)$ is a closed orbit; hence $x\left(t, x_{1}, \epsilon\right)$ is a closed orbit. $\quad$ Q.E.D.

We are now in a position to prove Theorem 1.1.

Proof of Theorem 1.1. Consider Eq. (1.1) written in the canonical form (1.3). Let $\epsilon_{1}$ be as in Theorem 3.4, let $r_{1}, r_{2}$, and $r(\epsilon), 0<\epsilon \leqslant \epsilon_{1}$, be as in Theorem 2.3. We will first prove the existence of the closed orbit $\gamma_{1}(\epsilon)$.

For any $\epsilon \in\left(0, \epsilon_{\mathrm{y}}\right]$ let $\rho(\epsilon)$ be as in Theorem 3.3 and let $x\left(t, x_{0}, \epsilon\right)$ be a nonzero solution of (1.3) with $x_{0} \in M^{2}(\epsilon) \cap B^{n}(\rho(\epsilon))$. Then, by Corollary 3.3.1 $x\left(t, x_{0}, \epsilon\right) \nrightarrow 0$ as $t \rightarrow+\infty$. Hence, by Theorems 3.2 and 3.3, $x\left(t, x_{0}, \epsilon\right)$ has a nonempty positive-limiting set $\omega^{+}$in $M^{2}(\epsilon) \cap\left[B^{n}(r(\epsilon))-B^{n}(\rho(\epsilon))\right]$ and $x\left(t, x_{0}, \epsilon\right) \rightarrow \omega^{+}$as $t \rightarrow+\infty$. By the final assertion of Theorem 3.4, $\omega^{+}$is a single closed orbit of (1.3) and, regarded as a closed Jordan curve in $M^{2}(\epsilon), \omega^{+}$contains the origin $x=0$ in its interior.

Now, if $x\left(t, \bar{x}_{0}, \epsilon\right)$ is any non-zero solution of (1.3) such that $\tilde{x}_{0} \in M^{2}(\epsilon)$ and such that $\tilde{x}_{0}$ is inside $\omega^{+}$, then by repeating the above arguments we can 
show that as $t \rightarrow+\infty x\left(t, \tilde{x}_{0}, \epsilon\right)$ approaches a single closed orbit $\bar{\omega}^{+}$in $M^{2}(\epsilon) \cap\left[B^{n}(r(\epsilon))-B^{n}(\rho(\epsilon))\right]$ and that $\bar{\omega}^{+}$contains the origin $x=0$ in its interior. Since $! x_{0}:<\rho(\epsilon)$ it follows from Corollary 2.3.1 that $\bar{\omega}^{+}=\omega^{+}$. We now let $\gamma_{1}(\epsilon)=\omega^{-}$. The preceding argument shows that any nonzero solution of (1.3) lying in $M^{2}(c)$ and inside $\gamma_{1}(\epsilon)$ must approach $\gamma_{1}(\epsilon)$ as $t \rightarrow+\infty$. We next show that such solutions approach the origin $x=0$ as $t \rightarrow-\infty$.

Consider again the solution $x\left(t, \bar{x}_{0}, \epsilon\right)$. This solution must remain in $M^{2}(\epsilon)$ and inside $\gamma_{1}(\epsilon)$ for all $t<0$. By the choice of $\epsilon_{1}$ in Theorem 3.4, the origin $x=0$ is the only equilibrium point of (1.3) inside $B^{n}(r(\epsilon))$. Hence, by Corollaries 2.3.1 and 2.3.2 and Bendixson's Theorem ([9], p. 230), the following is true: as $t \rightarrow-\infty x\left(t, \bar{x}_{0}, \epsilon\right)$ approaches either the origin $x=0$ or a closed invariant curve $\Gamma$ lying in $M^{2}(\epsilon)$ and inside $\gamma_{1}(\epsilon)$. If the latter case holds then solutions of (1.3) lying in $M^{2}(\epsilon)$ and inside $\Gamma$ cannot approach $\gamma_{1}(\epsilon)$ as $t \rightarrow+\infty$, and this contradicts the stability property of $\gamma_{1}(\epsilon)$ obtained above. Therefore, $x\left(t, \bar{x}_{0}, \epsilon\right) \rightarrow 0$ as $t \rightarrow-\infty$. This shows that any nonzero solution of (1.3) lying in $M^{2}(\epsilon)$ and inside $\gamma_{1}(\epsilon)$ must approach the origin $x=0$ as $t \rightarrow-\infty$. From Assertion (ii) of Theorem 2.3 it is clear that no other solution of (1.3) can have this property. Thus, (ii) of Theorem 1.1 is proved.

The proof of the existence of $\gamma_{2}(\epsilon)$ and of its stability property ((iii) of Theorem 1.1) is analogous to arguments given abovc, the only differcnce being that one starts with a solution $x\left(t, x_{0}, \epsilon\right)$ for which $x_{0} \in M^{2}(\epsilon) \cap$ $\left[B^{n}\left(r_{2}\right)-B^{n}(r(\epsilon))\right]$. We will omit the details.

Assertion (iv) of Theorem 1.1 follows directly from Theorem 3.1. It only remains now to establish assertion (v).

For any $\epsilon \in\left(0, \epsilon_{1}\right]$ let $\Omega(\epsilon)$ be the closed invariant set in $M^{2}(\epsilon)$ consisting of points on or between $\gamma_{1}(\epsilon)$ and $\gamma_{2}(\epsilon)$. 'Then suppose that $x\left(t, x_{0}, \epsilon\right)$ is a solution of (1.3) with $\mid x_{0} !<r_{2}$ and suppose that $x\left(t, x_{0}, \epsilon\right) \nrightarrow 0$ as $t \rightarrow+\infty$. By Theorems 3.2 and 3.3, $x\left(t, x_{0}, \epsilon\right) \mid<r_{1}$ for $t>0$ and $x\left(t, x_{0}, \epsilon\right)$ has a nonempty positive-limiting set $\omega^{+}$in $M^{2}(\epsilon) \cap\left[B^{n}(r(\epsilon))-B^{n}(\rho(\epsilon))\right]$; moreover, $x\left(t, x_{0}, \epsilon\right) \rightarrow \omega^{+}$as $t \rightarrow+\infty$. To prove (v) of Theorem 1.1 it suffices to show that $\omega^{+} \subset \Omega(\epsilon)$ and that $\omega^{+}$contains at least one closed orbit of (1.3).

Let $x_{1} \in \omega^{\perp}$ and suppose that $x_{1}$ lies inside $\gamma_{1}(\epsilon)$ on $M^{2}(\epsilon)$; then, $x\left(t, x_{1}, \epsilon\right)$ lies inside $\gamma_{1}(\epsilon)$. Therefore, by (ii) of Theorem $1.1 x\left(t, x_{1}, \epsilon\right)$ cannot be a closed orbit. Hence, by Theorem 3.4 there exist two distinct closed orbits $\Gamma^{+}$and $\Gamma^{-}$of $(1.3)$ in $M^{2}(\epsilon)$ concentric about the origin $x=0$, and $x\left(t, x_{1}, \epsilon\right) \rightarrow \Gamma^{+}$as $t \rightarrow+\infty$ and $x\left(t, x_{1}, \epsilon\right) \rightarrow \Gamma^{-}$. But, by (ii) of Theorem $1.1, x\left(t, x_{1}, \epsilon\right) \rightarrow \gamma_{1}(\epsilon)$ as $t \rightarrow+\infty$; hence, $\Gamma^{+}-\gamma_{1}(\epsilon)$ and, therefore, $\Gamma^{-} \neq \gamma_{1}(\epsilon)$; i.e., $\Gamma^{-}$is a closed orbit inside $\gamma_{1}(\epsilon)$ on $M^{2}(\epsilon)$. This contradicts (ii) of Theorem 1.1. Thus, we can not have $x_{1}$ inside $\gamma_{1}(\epsilon)$. Similar reasoning shows that $x_{1}$ can not be outside $\gamma_{2}(\epsilon)$. It follows, that $x_{1} \in \Omega(\epsilon)$, i.c., $\omega^{+} C \Omega(\epsilon)$. 
Now let $x\left(t, x_{2}, \epsilon\right)$ be any solution of $(1.3)$ in $\omega^{+}$. Then, by Theorem 3.4 its positive-limiting set is a closed orbit $\Gamma$. But then $\Gamma$ itself is contained in $\omega^{+}$. The proof is complete.

\section{ACKNOWLEDGMENT}

The author is indebted to Professor Jack K. Hale of Brown University for his advice and encouragement in the preparation of this work.

\section{REFERENCES}

1. Andronov, A. A., And Chaikin, C. E., "Theory of Oscillations." (S. Lefschetz, ed.) Princeton Univ. Press, Princeton, N. J., 1949.

2. Bogoliubov, N. N., And Mitropolsky, Y. A., "Asymptotic Methods in the Theory of Non-Linear Oscillations." Chapter 6. Hindustan Publishing Corp. (India), 1961. (Translated from Russian.)

3. Brushinskaya, N. N., Qualitative integration of a system of $n$ differential equations in a region containing a singular point and a limit cycle. Dokl. Akad. Nauk SSSR, 139 (1961), 9-12. MR 26 \# 5212 (1963). (In Russian.)

4. Coddington, E. A., And Levinson, N., "Theory of Ordinary Differential Equations." McGraw-Hill, New York, 1955.

5. HaHN, W., "Theory and Application of Liapunov's Direct Method." PrenticeHall, Englewood Cliffs, N. J., 1963.

6. HaLe, J. K., "Oscillations in Nonlinear Systems." McGraw-Hill, New York, 1963.

7. Hopf, E., Abzweigung einer Periodischen Lösung von einer Stationären Lösung eines Differential Systems. Ber. Verh. Sachs. Akad. Wiss. Leipsig. Math.-Nat. 94 (1942), 3-22.

8. LaSalle, J. P., AND Lefschetz, S., "Stability by Lipaunov's Direct Method with Applications." Academic Press, New York, 1961.

9. Lefschetz, S. "Differential Equations: Geometric Theory." Second Edition. Wiley, Interscience, New York, 1963.

10. Likova, O. B., On the behavior of solutions of a system of differential equations in the neighborhood of an isolated constant solution. Ukrain. Mat. Zh. IX (1957), 281-295. (In Russian.)

11. Likova, O. B., On the behavior of solutions of a system of differential equations in the neighborhood of closed orbits. Ukrain. Mat. Zh. IX (1957), 419-431. (In Russian.) 